\title{
CLOSE RANGE HYPERSPECTRAL IMAGING INTEGRATED WITH TERRESTRIAL LIDAR SCANNING APPLIED TO ROCK CHARACTERISATION AT CENTIMETRE SCALE
}

\author{
T. H. Kurz ${ }^{\text {a, }}$, S. J. Buckley ${ }^{\text {a }}$ J. A. Howell ${ }^{\text {a }}$ \\ ${ }^{\text {a }}$ Centre for Integrated Petroleum Research (Uni CIPR), Postboks 7810, 5020 Bergen, Norway - tobias.kurz@uni.no, \\ simon.buckley@uni.no,john.howell@uni.no
}

TS V/6: Close Range Morphological Measurement for the Earth Sciences

KEY WORDS: Geology, Hyperspectral, LIDAR, Close Range, High resolution, TLS

\begin{abstract}
:
Compact and lightweight hyperspectral imagers allow the application of close range hyperspectral imaging with a ground based scanning setup for geological fieldwork. Using such a scanning setup, steep cliff sections and quarry walls can be scanned with a more appropriate viewing direction and a higher image resolution than from airborne and spaceborne platforms. Integration of the hyperspectral imagery with terrestrial lidar scanning provides the hyperspectral information in a georeferenced framework and enables measurement at centimetre scale. In this paper, three geological case studies are used to demonstrate the potential of this method for rock characterisation. Two case studies are applied to carbonate quarries where mapping of different limestone and dolomite types was required, as well as measurements of faults and layer thicknesses from inaccessible parts of the quarries. The third case study demonstrates the method using artificial lighting, applied in a subsurface scanning scenario where solar radiation cannot be utilised.
\end{abstract}

\section{INTRODUCTION}

In recent years a major effort has been made to develop imaging methods to assist the description of rock properties, for application to hydrocarbon reservoirs, aquifers or the study of natural fluid barriers in host rocks. As the distribution of mineralogy and lithology with different geophysical properties, such as porosity and permeability, controls fluid flow or sealing properties, the heterogeneity of rocks is the focus of many geosciences studies. Furthermore, in many applications, correlation between the lithology and fault and fracture systems is required. To characterise the heterogeneity of rocks, different scales need to be integrated, from sub-millimetre to kilometre scale. However, appropriate imaging methods that connect the millimetre to metre scale are a missing link in many geosciences studies. Ground based hyperspectral imaging is a new technique in remote sensing, where a lightweight hyperspectral imager mounted on a tripod is used to map mineral variation in vertically-oriented rock faces, at high resolution. (Kurz et al. 2012). To relate the image to a real world coordinate system, the hyperspectral imagery is integrated with terrestrial laser scanning (TLS) (Kurz et al. 2011). The data integration adds geometric information, which may be used for hyperspectral image corrections, for verifying classification results and for analysing the distribution and content of the mineralogy and lithology.

This paper describes the general workflow of close range hyperspectral scanning, and demonstrates the potential using example data. Case studies from different locations and with different mineralogy are used to demonstrate the potential of hyperspectral imaging to describe rock characteristics in a noncontact manner, and at various scales. An example from a carbonate quarry in northern Spain shows that different dolomite and limestone types, which have very similar spectral properties, can be mapped and the portion of the different lithology could be quantified. In a quarry in Switzerland with inaccessible walls, 3-20 cm thick layers of interbedded carbonate and shale have been mapped, and fault displacements established using the combined lidar data and hyperspectral classifications. The method has also been tested in an underground rock laboratory in Switzerland, using an artificial light source.

\section{INSTRUMENTATION}

\subsection{Hyperspectral Imager}

The data from the case studies was collected with two different instruments, the HySpex SWIR-320m and the HySpex SWIR$320 \mathrm{me}$, both from Norsk Elektro Optikk. The latter provides an extended spectral range indicated by the "e" in the instrument name. The HySpex SWIR-320m collects 240 spectral bands with spectral sampling of c. $5 \mathrm{~nm}$ and operates within the short wave infrared (SWIR) spectral range between 1.3-2.5 $\mu \mathrm{m}$ (Table 1). The sensor with the extended spectral range operates between $1.0-2.5 \mu \mathrm{m}$ and collects 256 bands with a spectral sampling of c. $6 \mathrm{~nm}$. All other specifications are identical for both sensors (Table 1). Calibrated reflection targets $(32 \times 32$ $\mathrm{cm}$ ) with $50 \%$ and $99 \%$ reflectance, consisting of Spectralon

\footnotetext{
* Corresponding author
} 
material, were placed in the scanned scene and used for atmospheric image correction (Figure 1).

The instruments are line (pushbroom) sensors with 320 pixels in the scanning line. In a ground based setup (Figure 1), the sensors are mounted to a rotation stage and the sensor line is rotated to build up an image. The rotation results in panoramic image geometry, which must be considered for photogrammetric processing. The sensor optics have a field of view (FOV) of $14^{\circ}$ in the vertical direction. This FOV is relatively small and therefore often requires multiple images to be acquired to cover a high cliff section at short range. At $50 \mathrm{~m}$ range, a collected image has an object pixel size of $3.5 \mathrm{~cm}$.

As these sensors operate in the SWIR spectral range they are particularly suitable for geological applications, because many rock-forming minerals show diagnostic absorption features solely within this spectral range (Hunt 1980). These compact and lightweight instruments are highly portable, which is an important criterion for geological applications, since many field sites are not accessible by motor vehicles.

Table 1. Specifications of the HySpex SWIR-320m imager

\begin{tabular}{ll}
\hline Detector & $\mathrm{HgCdTe}(320 \times 256$ pixels $)$ \\
Spectral range & $1.3-2.5 \mu \mathrm{m}$ \\
Spectral sampling & $\sim 5 \mathrm{~nm}$ \\
Number of bands & 240 \\
Digitisation & $14 \mathrm{bit}$ \\
Spatial pixels in the vertical line & 320 \\
Field of view across track & $14^{\circ}$ \\
\hline
\end{tabular}

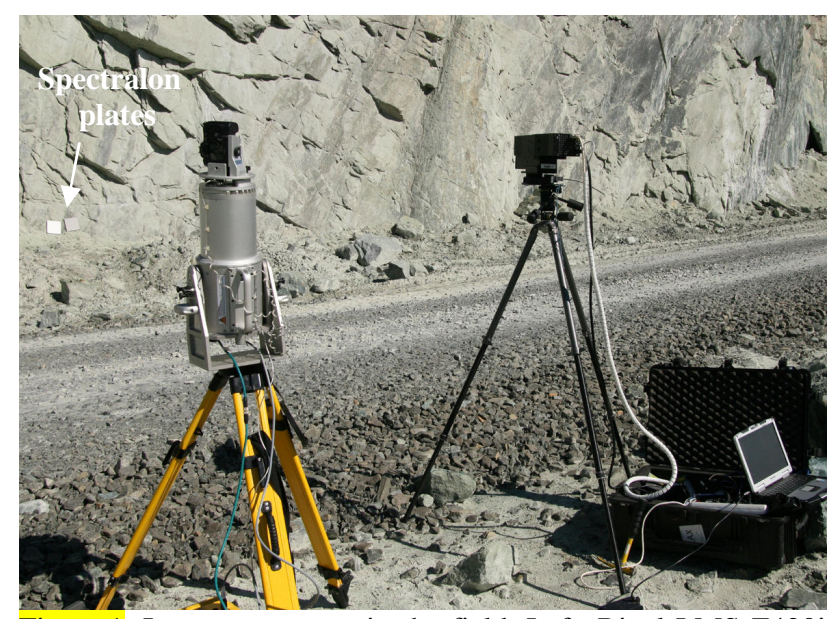

Figure 1. Instrument setup in the field. Left: Riegl LMS-Z420i scanning system with a digital camera rigidly mounted on top of the sensor; Right: HySpex SWIR-320m camera mounted on a rotation stage.

\subsection{Terrestrial Laser System}

The lidar data were collected with a Riegl LMS-Z420i instrument (Figure 1). This system is designed for medium to long range scanning (up to $800 \mathrm{~m}$ for high reflectance targets), and has a quoted point precision of $0.01 \mathrm{~m}$. A calibrated Nikon D200 camera (10 megapixels) was rigidly mounted on top of the laser scanner, allowing registered imagery to be acquired for later photorealistic modelling. Photos were acquired with calibrated $50 \mathrm{~mm}$ and $85 \mathrm{~mm}$ Nikkor lenses.

\section{METHOD}

\subsection{Hyperspectral Data Processing}

Empirical Line (Smith and Milton, 1999) correction was applied using the Spectralon targets, to remove atmospheric effects and to transfer the radiance images into reflectance images. All hyperspectral images are affected by artefacts such as striping, brightness gradients and bad pixels. Most of these image artefacts are non-conformities typically associated with pushbroom sensors (Nieke et al. 2008), though they are increased due to the panoramic image geometry and the ground based scanning setup, which introduces topographic effects. Different correction approaches, such as polynomial interpolation to remove brightness gradients and filtering in the frequency domain to remove striping, were applied.

Maximum Noise Fraction transformation (MNF), a modification of the principal component analysis for hyperspectral data, was used to remove random noise and to reduce the data dimensionality (Green et al., 1988). MNF images, which visualise noise-free MNF bands in false colour RGB (red, green, blue), are also frequently used for preliminary image interpretation.

Collecting end-member spectra is the first stage of classification, as understanding end-member signatures allows the content of material within mixed pixels to be estimated. End-members are spectra from spectrally pure materials (i.e. a pixel that measures a single material) and are used as classification input. In this work, end-member spectra have been extracted either from areas with known mineralogy or using statistical methods as described in (Kruse et al., 2003).

Different approaches have been applied to create hyperspectral classification maps, such as band ratioing, Spectral Angle Mapper (SAM; Kruse et al., 1993) and Mixture Tuned Matched Filtering (MTMF; Boardman, 1998). Classification results were quantitatively checked by comparison with the higher resolution photorealistic lidar models for classes that could be mapped from the conventional photographs.

\subsection{Terrestrial Laser Data}

The lidar data processing has the purpose of generating a photorealistic lidar model, as described in Buckley et al. (2008 and 2010), which allows geometric measurements and geological interpretations to be made. To ensure complete coverage of a field site, and to avoid scan shadows, multiple scan positions are required with an overlap of at least $20 \%$. The registration of the separate lidar scans is carried out using a shape fitting algorithm. Subsequently, the merged point cloud is cleaned of vegetation, as well as foreground and background material not relevant to the geology. The point cloud is than meshed by fitting a triangular surface to the $3 \mathrm{D}$ points. Finally the meshed surface is textured with the high resolution photographs. Since the digital camera is rigidly mounted to the laser scanner, the photographs are registered within the lidar coordinate system automatically. Lens distortion is removed from the images by resampling prior to texture mapping. 


\subsection{Data Integration}

In contrast to frame images, the hyperspectral imagery has to be processed with a panoramic camera model to determine the image orientation within the lidar coordinate system (Kurz et al., 2011). A linear-array-based terrestrial panoramic camera model with additional correction parameters, as described in Schneider and Maas (2006), is applied. In this camera model the panoramic image geometry is described using a cylindrical projection. Exterior and interior orientation of the hyperspectral images are than determined based on block bundle adjustment (Kurz et el., 2011).

The meshed lidar surface can be subsequently textured with hyperspectral image products. Texturing of the lidar models with multiple different hyperspectral products, such as classification and MNF images, allows interactive evaluation and interpretation of results. Geometric information of the lidar models, such as slope, aspect and size of each image pixels can be exported and used to improve the processing of the hyperspectral imagery.

\section{CASE STUDY: POZALAGUA QUARRY}

At the Pozalagua Quarry (Cantabrian Mountains, Spain), Cretaceous carbonates of a carbonate platform slope are exposed. The host limestone has been partly dolomitised due to fault-related hydrothermal alteration processes. The geological history and the digenesis are presented in detailed in Swennen et al. (in press). The quarry has been reshaped for use as an amphitheatre and provides both weathered and fresh-cut walls, which are ideal for detailed studies of dolomitisation processes. The transformation of limestone into dolomite, due to alteration processes, results often in an increased porosity and can therefore significantly improve the properties of hydrocarbon reservoirs in carbonates. Since subsurface reservoirs allow only limited access (bore holes, seismic data), geophysical properties and geological processes are studied in detailed, at metre to centimetre scale, using so called outcrop analogues (such as the Pozalagua Quarry). However, methods are required that allow the mapping as well as the quantification of the mineralogy and lithology even in inaccessible parts of the quarry walls.

In this case study, ground based hyperspectral imaging integrated with TLS is used to separate limestone and dolomite and to map the distribution and quantify different dolomite generations. Carbonate minerals show pronounced absorption features within the SWIR spectral range, due to vibrational processes of carbonate ions within the crystal lattice (Clark, 1990). However, the higher manganese content of dolomite results in a slight shifting of the carbonate absorption features to lower wavelengths, allowing the discrimination of these materials with high reliability using hyperspectral imaging (Van der Meer, 1995).

Hyperspectral data was collected with the HySpex SWIR-320m sensor. Figure 2 shows the end-member spectra of the different mineralogy in the Pozalagua quarry. As well as limestone and dolomite it is also possible to spectrally differentiate between different limestone and dolomite types even though these materials are chemically very similar. In the limestone spectra, different organic content modifies the intensity of the carbonate absorption features. The zebra dolomite contains more iron and manganese compared to the coarse crystalline dolomite that results in a flattened carbonate absorption feature at $2150 \mathrm{~nm}$ (Kurz et al., 2012).

Figure 3 shows a result of the hyperspectral mapping. The bluish and reddish colours (Figure 3B) indicate a sharp border between limestone and dolomite, as well as material differences that are not recognisable in visible light (yellow arrows in Figure 3B). Using the MTMF classification approach the mineralogy could be reliably mapped also in inaccessible parts of the quarry. Since the hyperspectral map is integrated with the lidar data and therefore georeferenced, it is also possible to quantify the mineralogy and make volume estimations. The organic rich limestone, representing cave infill material, appears in visible light very similar to the surrounding limestone and has been therefore overlooked during previous fieldwork; however this material can be mapped using hyperspectral imaging.

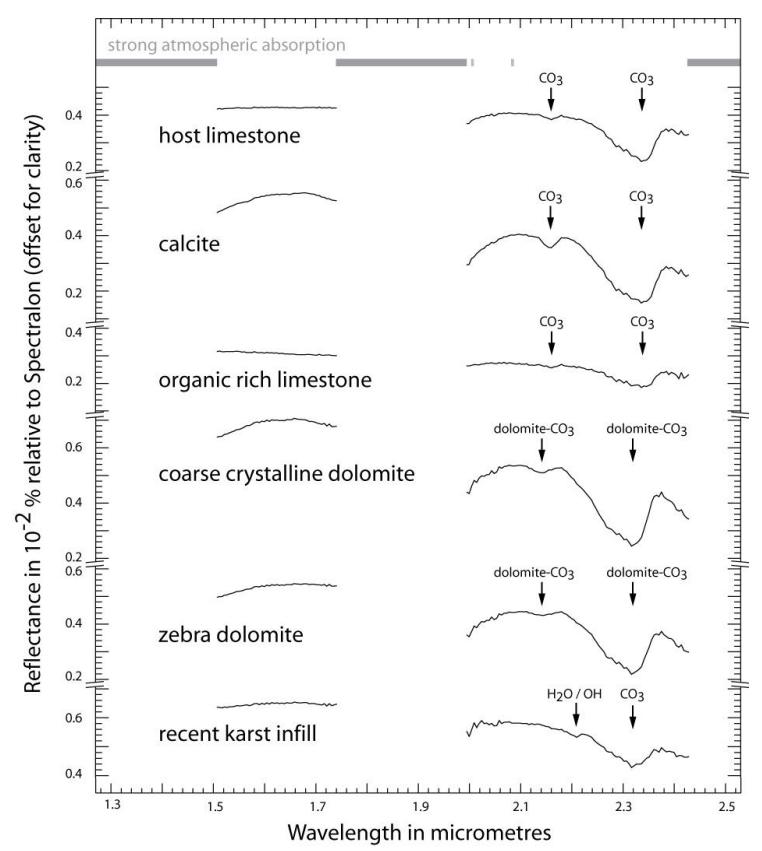

Figure 2. End-member spectra from the Pozalagua quarry, data collected with the HySpex SWIR-320m sensor (after Kurz et al., 2011). 

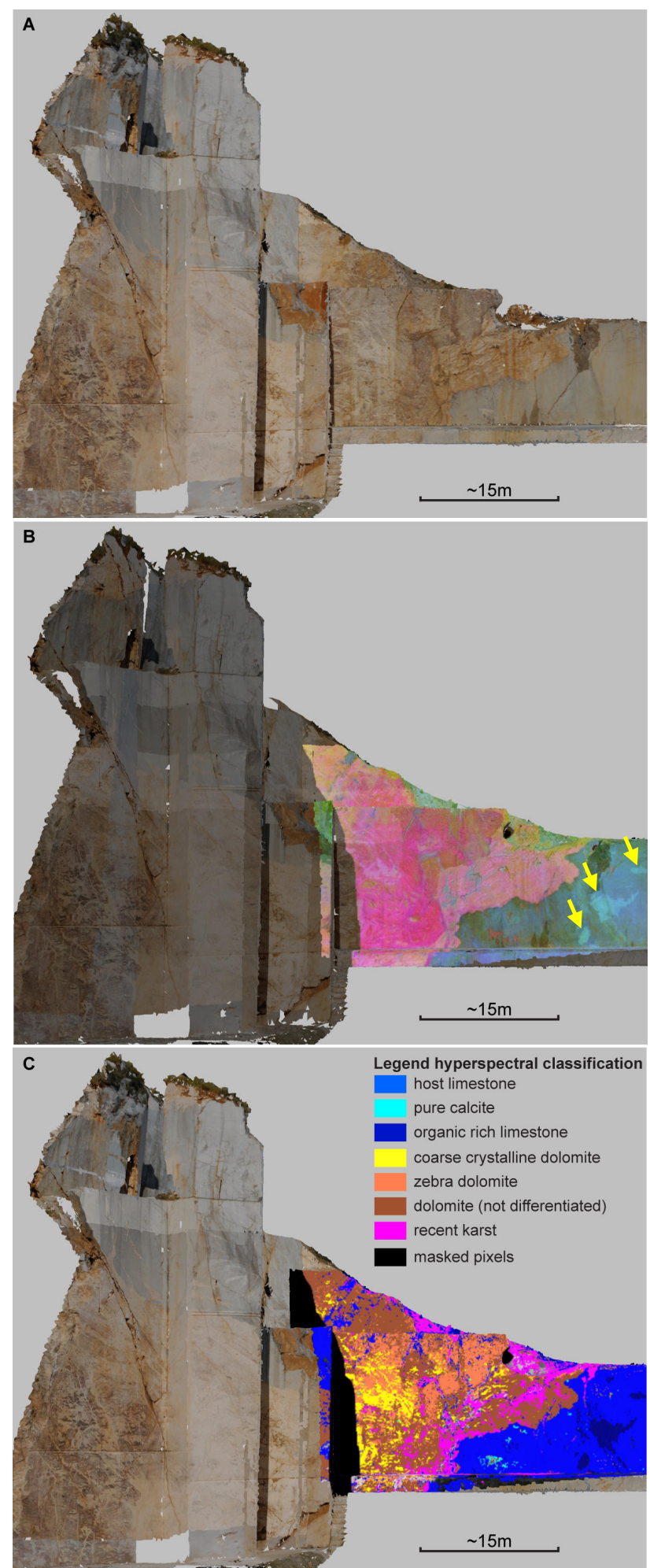

Figure 3. Results from the Pozalagua quarry: photorealistic lidar model (A) textured with B) MNF image; C) a hyperspectral classification (MTMF classification).

\section{CASE STUDY: JAKOBSBERG QUARRY}

In the Jakobsberg Quarry (near Wildegg, Switzerland), interbedded marl, carbonates and shales (Effingen Member, Malm, Jura) are exposed. The quarry walls are up to $50 \mathrm{~m}$ high and very unstable, requiring remote mapping methods. The aim of this study was to map the carbonate and shale layers, determine layer thicknesses and measure fault displacements. For this case study the HySpex SWIR-320me sensor was used.

Figure 4 shows a preliminary result of the hyperspectral mapping, where bluish colours indicate shale material and reddish colours highlight carbonates. The interbedding is clearly visible in the MNF image of Figure 4B. Utilising the lidar geometric data, layer thicknesses and fault displacements can be measured and faults can be mapped and quantified (Figure 5).

The hyperspectral images at Jakobsberg exhibited a high noise level, hampering the analysis of pixel spectra. The high noise level results partly from poor illumination conditions, as northfacing exposures meant it was difficult to image this cliff face with illumination from behind. Another reason for the high noise level is related to the extended spectral range of the HySpex SWIR-320me sensor. In the field, the exposure time of an image was adjusted so that the spectrally most sensitive bands, appearing around $1000 \mathrm{~nm}$ for the HySpex SWIR-320me sensor, exploited the full data range (14 bit) and avoiding oversaturation. In contrast, the bands of the spectral range between 2000-2500, most relevant for carbonate and hydroxyl absorption features, used less than $20 \%$ of the dynamic data range. This dynamic range problem made if difficult to study the clay and carbonate material in detail.

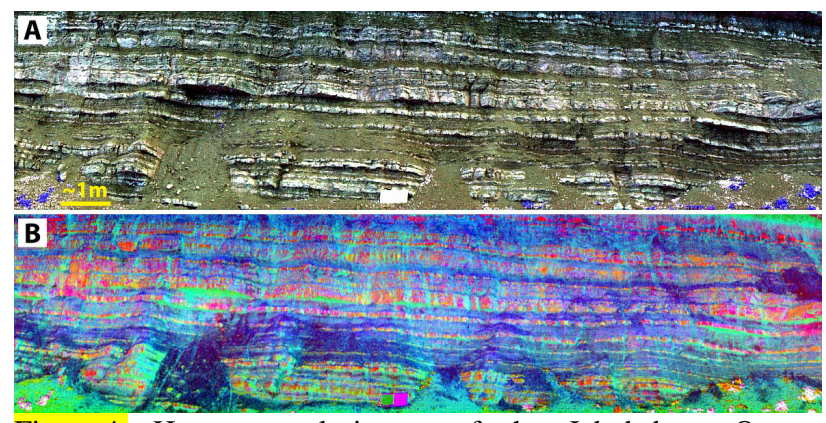

Figure 4. Hyperspectral image of the Jakobsberg Quarry collected with the HySpex SWIR-320me sensor; A: reflectance image as false colour (bands $1442 \mathrm{~nm}$, $2164 \mathrm{~nm}$ and $1249 \mathrm{~nm}$ shown in RGB); B: MNF image, (bands 5, 6 and 7 shown in RGB).

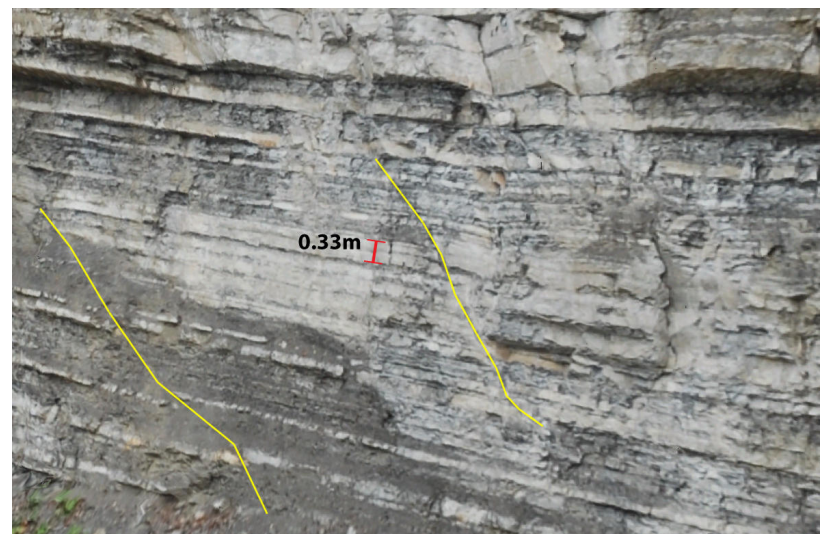

Figure 5. Photorealistic lidar model from the Jakobsberg Quarry with fault mapping (yellow lines) and thickness measurement of one carbonate layer (red). 


\section{CASE STUDY: ROCK LABORATORY}

In some scenarios, such as in tunnels or subsurface mining, it is not possible to use solar radiation though remote mineral mapping at centimetre to metre scale is still desired to allow efficient data collection and to ensure staff safety. The subsurface rock laboratory at Mont Terri (Jura Mountains, Switzerland) is used to develop instruments and research methods for hydrogeological, geochemical and geotechnical characterisation of argillaceous formations. The formation of interest in the rock laboratory is the Opalinus Clay, a claystone of Jurassic age consisting mainly of the clay minerals kaolinite, illite, smectite and chlorite, as well as impurities such as quartz, carbonates and feldspars (Corkum and Martin, 2007). Part of this setting, the TT Niche, was used to assess the applicability of ground based hyperspectral imaging with artificial lighting, and to test the potential of this method for detailed characterisation of the Opalinus Clay.

For the purpose of the feasibility study, a low cost solution for the artificial lighting was chosen. Three $500 \mathrm{~W}$ halogen lamps and two $2000 \mathrm{~W}$ non-glare balloon lamps, commonly used at construction sites, were used. This configuration ensured satisfactorily uniform illumination (Figure 6). Halogen lamps emit visible and infrared light and provide therefore an appropriate light source for imaging in the SWIR spectral range. The data were collected with the HySpex SWIR-320me sensor. Figure 7 shows a hyperspectral image acquired with a scanning range of $13 \mathrm{~m}$, resulting in an image pixel resolution of $\mathrm{c} .1 \mathrm{~cm}$.

Figure 7 shows a hyperspectral result integrated with the photorealistic lidar model of the TT Niche. Some layers in the Opalinus Clay are clearly visible with reddish colours in the MNF image. These layers are not visible to the naked eye, and were missed with conventional mapping. Spectral signatures from these areas show carbonate absorption features (Figure 8) and allow therefore a reliable mapping of these carbonatebearing clay layers using hyperspectral imaging.

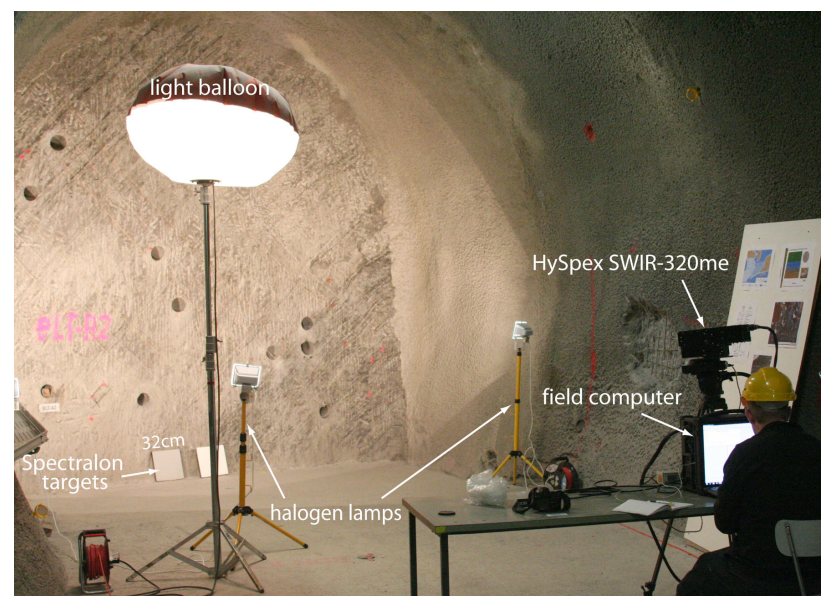

Figure 6. Scanning setup in the subsurface rock laboratory.

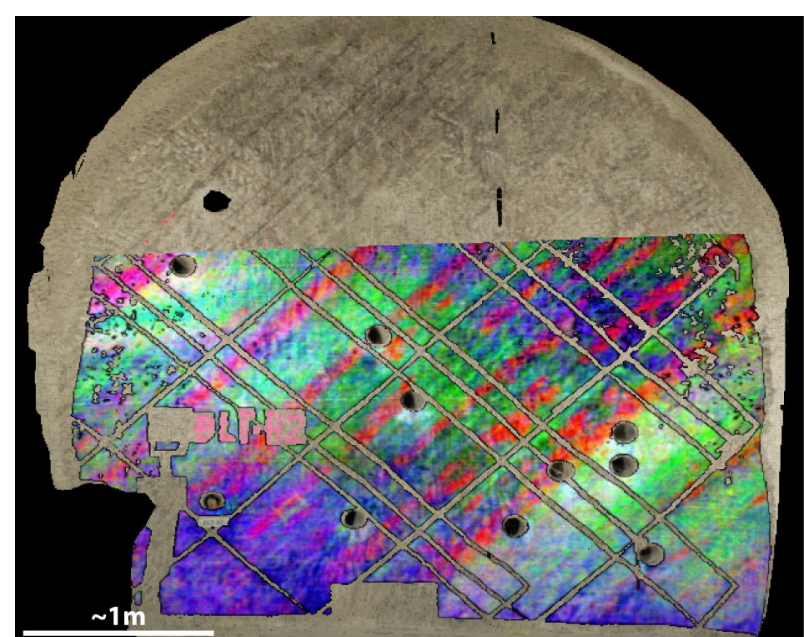

Figure 7. Result from the TT Niche; photorealistic lidar model multi-textured with a hyperspectral image product (MNF image, bands 5, 6 and 7 visualised in RGB); red colours highlight carbonate-bearing material.

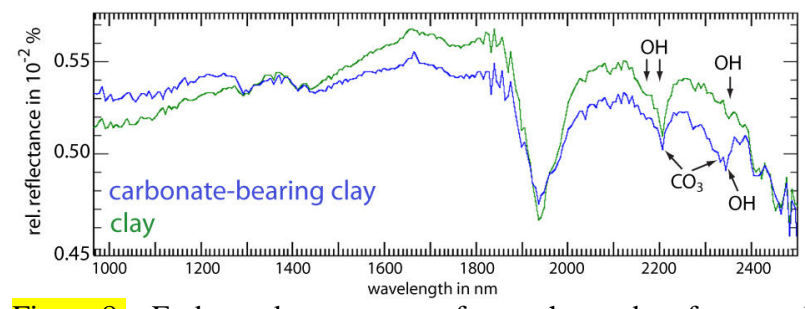

Figure 8. End-member spectra from the subsurface rock laboratory. Carbonate absorption features allow the identification of carbonate-bearing clay; data collected with the HySpex SWIR-320me sensor.

\section{CONCLUSIONS}

The use of ground based hyperspectral imaging has the potential to complement a number of applications where information on the spatial distribution of materials is required, at a variety of scales. The hyperspectral image correction can be improved by utilising the geometric information determined from the lidar data. The additional tight coupling with terrestrial laser scanning data gives the possibility to exploit hyperspectral results in an accurate and quantitative way. More research is required to develop correction methods for the hyperspectral imagery adjusted to the panoramic image geometry and the ground based scanning setup. The case study of the subsurface rock laboratory demonstrates the applicability of artificial light sources for applications where solar radiation cannot be employed. It is concluded that ground based hyperspectral imaging will become a standard method in many geosciences applications.

\section{References}

Boardman, J., 1998, Leveraging the high dimensionality of AVIRIS data for improved sub-pixel target unmixing and rejection of false positives: Mixture Tuned Matched Filtering. In 7th Annual JPL Airborne Geoscience Workshop, 1998, Pasadena, CA, pp. 55-56. 
Buckley, S.J., Howell, J.A., Enge, H.D., Kurz, T.H., 2008. Terrestrial laser scanning in geology: data acquisition, processing and accuracy considerations. Journal of the Geological Society, 165(3), pp. 625-638.

Buckley, S.J., Enge, H.D., Carlsson, C., Howell, J.A., 2010. Terrestrial laser scanning for use in virtual outcrop geology. The Photogrammetric Record, 25(131), pp. 225-239.

Clark, R.N., King, T.V.V., Klejwa, M., Swayze, G. A., and Vergo, N., 1990. High spectral resolution reflectance spectroscopy of minerals. Journal of Geophysical Research, 95, pp. $12653-12680$.

Corkum, A.G., Martin, C.D., 2007. The mechanical behaviour of weak mudstone (Opalinus Clay) at low stresses. International Journal of Rock Mechanics \& Mining Sciences, 44, pp. 196-209

Green A. A., Berman M., Switzer P., Craig M. D., 1988. A transformation for ordering multispectral data in terms of image quality with implications for noise removal. IEEE Transactions on Geoscience and Remote Sensing, 26, pp. 65-74.

Hunt, G.R., 1980. Electromagnetic radiation; the communication link in remote sensing. In: B. S. Siegal, B.S., Gillespie, A.R. (Eds.) Remote Sensing in Geology, John Wiley \& Sons, New York, p. 5-45.

Kruse, F.A., Lefkoff, A.B., Boardman, J.B., Heidebrecht, K.B., Shapiro, A.T., Barloon, P.J. and Goetz, A.F.H. (1993) The Spectral Image Processing System (SIPS) - Interactive Visualization and Analysis of Imaging spectrometer Data. Remote Sensing of Environment, 44, 145-163.

Kruse, F.A., Boardman, J.W. and Huntington, J.F. (2003) Comparison of Airborne Hyperspectral Data and EO-1 Hyperion for Mineral Mapping. IEEE Transactions on Geoscience and Remote Sensing, Special Issue, 41(6), pp. 1388-1400.

Kurz, T.H., Buckley, S.J., Howell, J.A., Schneider, D., 2011. Integration of panoramic hyperspectral imaging with terrestrial lidar. Photogrammetric Record, 26(134), pp. 212-228.

Kurz, T.H., Dewit, J., Buckley, S.J., Thurmond, J.B., Hunt, D.W. and Swennen, R., 2012: Hyperspectral image analysis of different carbonate lithologies (limestone, karst, hydrothermal dolomites): the Pozalagua Quarry case study (Cantabria, NW Spain). Sedimentology, 59(2), pp. 623-645.

Nieke, J., Schläpfer, D., Dell'endice, F., Brazile, J., and Itten, K. I., 2008. Uniformity of imaging spectrometry data products. IEEE Transactions on Geoscience and Remote Sensing, 46, pp. 3326-3336.

Schneider, D., and Maas, H.-G., 2006, A geometric model for linear-array-based terrestrial panoramic cameras. The Photogrammetric Record, 21, pp. 198-210.

Smith M.G., Milton J.E., 1999. The use of the empirical line method to calibrate remotely sensed data to reflectance. International Journal of Remote Sensing, 20, pp. 2653-2662.

Swennen, R., Dewit, J., Fierens, E., Muchez, P., Nader, F., Shah, M., Hunt, D., in press. Multiple fluid flow dolomitisation events along the Ranero Fault (Pozalagua Quarry, Basque Cantabrian Basin): episodic earthquake activity. Sedimentology, published online: 31 Jan 2012, doi: 10.1111/j.13653091.2011.01309.x

Van der Meer, F., 1995. Spectral reflectance of carbonate mineral mixtures and bidirectional reflectance theory: quantitative analysis techniques for application in remote sensing. Remote Sensing Reviews, 13, pp. 67-94.

\section{Acknowledgements}

The Norwegian Research Council, Statoil ASA, the Mont Terri Consortium and NAGRA are thanked for financial support in the case studies. Norsk Elektro Optikk AS and Riegl GmbH are acknowledged for providing hardware and software support. Danilo Schneider is thanked for collaboration on the data integration. We would also like to thank Jens Becker from NAGRA for project organization and logistical support. Maria Krüger and Till Sieberth provided assistance with data collection at Mont Terri and the Jakobsberg Quarry. 